\title{
Una orientación propedéutica al másigt hablado en Melilla
}

José Antonio Valverde Martín, Halifa Kaddur Mohamed, Yamila Mohamed Maanan

Este artículo recoge uno de los muchos esquemas posibles para la iniciación en el conocimiento del másigt hablado en Melilla, un idioma que constituye una de las entidades culturales fundamentales de nuestra ciudad. Este esquema adopta un objetivo meramente propedéutico en dos sentidos: a) su ciclicidad, esto es, partiendo de unos presupuestos gramaticales y de un vocabulario mínimo se sientan unas bases (lo más adaptadas posibles al español en cuanto a escritura) para ampliarse cuantitativamente y cualitativamente; b) su orientación fundamental que alude a la ciclicidad pretendida en función de la oralidad y la reflexión a realizar por el lector.

\section{Advertencia preliminar}

Lector, vamos a ofrecerte un esquema de aprendizaje del másigt. Se trata de proporcionarte -e insistimos en esto- una estrategia para iniciarte en el estudio de esta lengua, vecina y desconocida del mundo español, y otro esquema para que tú desarrolles una labor de confeccionar, practicar y ampliar tus conocimientos del másigt. Para este segundo fin te ofrecemos un sistema de escritura y un modo de catalogar y componer verbos, oraciones, etc. Por tanto, si tú, lector, buscas una obra de gran profundidad gramatical por grandes conocimientos, vanidad 
o exceso de escrúpulo, no leas esto. Quizás sabes -o crees saberdemasiado. Esto es un esquema básico para aprender másigt. Si buscas un gran vocabulario en este modestísimo trabajo, entonces no lo leas, pero debes saber que si utilizas tus conocimientos de másigt para despreciar este trabajo estás levantando una barrera más (y ya son millones) entre nuestras dos culturas.

¿Realmente hay alguien cabal que piense que este modesto trabajo es un tratado másigt comparable a la gran obra del Padre Sarrionandía o los tratados de Laoust o Peregrín?

A ti, que no sabes el másigt y necesitas un primer paso para comenzar el camino de su conocimiento te ofrecemos este esquema de aprendizaje. Léelo y practícalo. Y cuando lo domines, lánzate sin miedo, para, usando los esquemas básicos gramaticales que te ofrecemos, ampliar, contextualizar y, en definitiva, aprender significativamente el másigt en un proceso empático y continuo, a tu ritmo y para tu uso. Así podremos conocernos, comprendernos y caminar juntos en un proyecto de vida común.

\section{Introducción}

Parece obvio que lo primero que hemos de plantearnos en este trabajo es el por qué hablar y comprender el másigt. Dos razones proponemos, razones que por otra parte nos han movido a la realización de este artículo:

a) Lenguaje y cultura son dos términos interactuantes, uno no puede entenderse sin el otro. Ast pues, adquirir una base de conocimiento sobre el lenguaje másigt nos va a posibilitar comprender la cultura bereber del Norte de Africa y concretamente la más próxima a nosotros, la de la Guelaia; comprensión provocada por la necesidad inequívoca de pensar en másigt para hablarlo. Para esto, lector te ofrecemos una base para que, como educador $y / 0$ autoeducador, des el primer paso para entender y hablar la masigta, llegando asi a dominarla.

b) Realmente resulta del todo innegable la presencia de la comunidad rifeña en nuestra ciudad. Debemos realizar ante esto un esfuerzo por comprender y colaborar para la mejora en todos los aspectos personales y colectivos de nuestra ciudad.

Es esta una oportunidad única para que las culturas española 
y la masigta se comprendan y colaboren en un marco único donde convivimos culturas tan distintas como la hindú, la cristiana, la musulmana y la judía.

En este artículo ofrecemos un trampolín (valga el símil) a partir del cual puedas zambullirte en la cultura masigta de Melilla. Este trabajo trata de servir al educador y al no educador a formarse y formar a otros dentro de un marco de educación multicultural en el que no se trata de buscar la asimilación de la cultura rifeña por la espanfola, sino de buscar y lograr la integración de la primera en la segunda y enriquecerse mutuamente.

En este trabajo puedes encontrar, lector, unos esquemas gramaticales, fonéticos y sintácticos del másigt que se habla en Melilla y la zona que la circunda. Queremos insistir en esto porque el másigt que vas a comenzar a aprender es una de las otras lenguas derivadas del Bereber que puede encontrarse en Marruecos, Argelia y otros países africanos; un másigt que ha ido cambiando con los años, a veces de forma vertiginosa, debido a que no se escribe $\mathrm{y}$, por tanto, ni se conserva, ni su gramática está sistematizada. Ante esta contrariedad hemos optado por utilizar caracteres latinos, aunque bien podrín haberse utilizado los árabes.

A nivel gramatical vamos a ofrecerte un esquema que contiene los siguientes puntos: formación del femenino; del plural; el adjetivo; el orden de la oración; el verbo; los pronombres personales y posesivos; las oraciones interrogativa y exclamativa; la negativa; el presente activo; los numerales y cardinales; y los adverbios. Es necesario decir desde un principio que estas reglas se cumplen en la generalidad del másigt, aunque existen multitud de excepciones propias de una lengua no escrita y, por tanto, no sistematizada.

A nivel fonético explicamos cómo deben leerse determinados caracteres y sonidos que no existen en el español y aclaramos las reglas de acentuación.

A continuación ofrecemos un vocabulario mínimo dispuesto en función de un esquema de aprendizaje en el que partiendo de verbos de uso cotidiano que actúan de centro de palabras referidas a ellos, se alcanzan verbos menos cotidianos, también como centros de otras palabras a partir de las que se hacen combinaciones más complejas.

El esquema se conforma y ejecuta siguiendo el modelo que a continuación proponemos, las caracteristicas de quien aprende y sus conocimientos previos. 
El esquema por tanto es el siguiente:

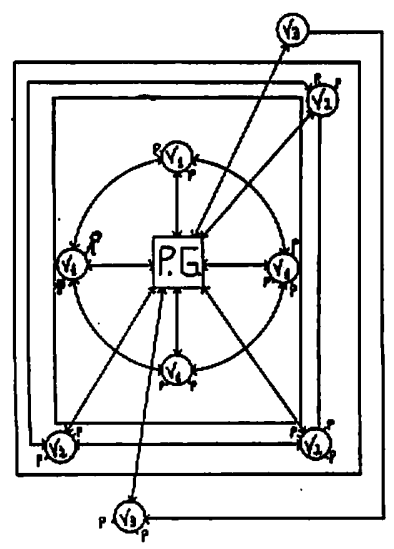

V1: verbos de uso cotidiano.

V2: verbos de uso poco cotidiano.

V3: verbos de uso apenas cotidiano.

P: palabras relacionadas con los verbos.

PG: principios gramaticales básicos.

Partiendo de los $\mathrm{Pg}$ podemos ir a los verbos y/o viceversa. Existe una interacción constante entre tipos de verbos:

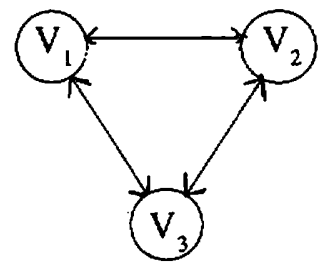

Este esquema está reflejado en la forma de disponer el vocabulario mínimo en el apartado cuarto.

El educador deberá, lógicamente, ajustarse a las peculiaridades de los educandos, personalizando en función de las evaluaciones inicial y formativa realizadas y considerar la edad y el momento psicoevolutivo del alumno de cara al proceso de enseñanza-aprendizaje, tomando en cuenta siempre que en la enseñanza-aprendizaje de toda lengua (y más en la masigta, lengua que actualmente no se escribe y está 
repleta de excepciones) hay que partir de la oralidad para después pasar a la escritura para, finalmente, cuando el educando haya alcanzado plenamente el periodo de las operaciones concretas iniciar unas líneas gramaticales muy elementales y relacionadas con la oralidad practicada y logicamente, el contexto.

A nivel de autoensenanza es necesario considerar los pasos propuestos en el esquema y la práctica continuada a nivel de oralidad.

Para terminar esta introducción es necesario aclarar de modo breve si el másigt es lengua o dialecto. Nosotros optamos por considerarlo lengua, ya que sus estructuras gramaticales y una parte de su vocabulario proceden de la antigua lengua bereber, aunque otra parte muy considerable (cuantitativa y cualitativamente hablando) de su vocabulario está contaminada por el árabe y el español. Por tanto el másigt no es una derivación de otra lengua (dialecto), sino una lengua que ha fundido elementos de otras lenguas.

\section{Aspectos gramaticales y fonéticos del másigt de Melilla}

Antes de comenzar tenemos que insistir en el carácter introductorio de esta gramática debido a las numerosas excepciones del másigt. Es necesario, por tanto, partiendo de la base gramatical y del vocabulario que proponemos, ajustar con la práctica oral lo aquí expuesto y ampliarlo cuantitativamente y cualitativamente.

\section{Aspectos gramaticales:}

A- Abecedario: señalamos aquí el abecedario que vamos a utilizar. Se explicará con más detenimiento en el aspecto fonético. s, sh, ç, t, th, u, x, y, z.

$a, a ̂, b, c, d, d h, e, f, g, g h, i, j, j h, k, 1, l h, m, n, o, p, q, r$,

B- Formación del femenino: Se realiza de dos formas:

1) $Z a+$ palabra.

Ejemplo: áharant $=$ niño; $z$ áharant $=$ niña

2) Za + palabra + sht: suele utilizarse cuando la palabra en masculino termina en vocal. 
J.A. Valverde, Halifa Kaddur. Yamila Mohamed

Ejemplo: arume $=$ cristiano; zarúmesht $=$ cristiana.

3) Hay conceptos para los que se utiliza una palabra en femenino y otra en masculino.

Ejemplo: Ariaç $=$ hombre: Zámegarth $=$ mujer

4) Hay palabras que no varian en cuanto al género: Ejemplo: Ueihere $=$ feo/a 0 malo/a.

\section{C- Eormación del plural:}

1) Para nombres masculinos se forma añadiendo a la palabra el prefijo $i$ y el sufijo $e$.

hombres

Ejemplo: Ariaç $=$ hombrc; $i+$ árią $+e n-$ iáriaçen $=$

Arume $=$ cristiano; $i+$ arume + en $=$ airumen $=$ cristianos

Muchas veces el prefijo $i$-absorbe la primera sflaba de la 96 palabra si ésta es vocal.

2) Para nombres femeninos se forma cambiando la silaba za inicial por $z i$ y añadiendo a la palabri cl sufijo en.

Ejemplo: Zámegarth $=$ mujer $=$ Zá-megarth

Zi-megar + th-en $=$ Zimegarthen $=$ mujeres

D) El adjetivo:

1) Se coloca siempre detrás del sustantivo.

Ejemplo: Ariaç ámkram = gran hombre

2) Existe una excepción cn los adjetivos "un, uno, una" que se colocan delante del sustantivo:

Ejemplo: Ixen áriac $=$ un hombre, ixet zámegarth $=$ una mujer

E- El orden de la oración: 

complementos.

El orden es siempre: sujeto (a veces se omite) + verbo +

Ejemplos: Nesh ghari ids $=$ Yo tengo sueño.

F) El verbo:

1) Normalmente en el másigt existen tres tiempos. Hay en el verbo dos formas, una para el presente, otra para el pasado. El futuro se forma añadiendo el prefijo ad- a la forma del presente. Su traducción es equivalente tanto a "voy a" mas infinitivo, como a nuestro futuro. Las terminaciones son:

Ejemplo: Snó = saber, conocer

Presente

Singular

Prefijo-Verbo-Sufijo

1) $\quad 1^{\mathrm{a}}$ persona

2) $2^{\mathrm{a}}$ persona

$-j$

1) Snoj

3.1) $3^{\mathrm{a}}$ persona (masc.) $i$ -

2) Snod

3.2.) $3^{\mathrm{a}}$ persona (fem.) -th

3.1.) Isnó

3.2.) Thsnó

Plural

4) $1^{\text {a }}$ persona

5.1) $2^{\mathrm{a}}$ persona (masc.) $i$ -

5.2.) $2^{\mathrm{a}}$ persona (fem.) th-

-em

4) Nesnó

6.1.) $3^{\mathrm{a}}$ persona (masc.)

-ent

5.1) Isnóem

6.2.) $3^{\mathrm{a}}$ persona (fem.)

-em

5.2.) Thsnóent

-ent

6.1.) Snóem

6.2.) Snóent

Pasado

En el pasado se utilizan las mismas terminaciones, pero la raíz cambia. Si no se conoce la forma del pretérito se puede añadir a la rafz del presente el prefijo togha-.

1) Togha-sno-j = toghasnoj

2) Togha-sno-d= toghasnod

3.1.) Togha-i-snó = toghaisnó 
J.A. Valverde, Halifa Kaddur, Yamila Mohamed

3.2.) Togha-th-snó = toghathsnó

4) Togha-ne-snó = toghanesnó

5.1.) Togha-i-snó-em = toghaisnóem

5.2.) Togha-th-snó-ent $=$ toghatsnóent

6.1.) Togha-snó-em $=$ toghasnóem

6.2.) Togha-snó-ent $=$ toghasnóent

Futuro: Para el futuro añadimos el prefijo ad-
1) $\quad$ Ad-sno-j $=$ Adsnoj
4) Ad-ne-snó $=$ Adnesnó
2) $A d$-sno-d $=$ Adsnod
5.1.) Ad-i-snó-em $=$ Adisnóem
3.1.) $A d-i$-snó $=$ Adisnó
5.2.) Ad-th-snó-ent $=$ Adtnsnóent
3-2) Ad-th-snó = Adthsnó
6.1.) Ad-snó-em $=$ Adsnóem
6.2) Ad-snó-ent $=$ Adsnóent

2) El participio se forma de la siguiente forma

$\begin{array}{ll}\text { Masculino: Singular: } i+\text { verbo } & \text { Plural: } i+\text { verbo }+e n \\ \text { Femenino: Singular: } t h+\text { verbo } & \text { Plural: } t h+\text { verbo }+e n\end{array}$

Masculino Singular: isnó

Femenino sing: thsnó
Plural: isnoen

Plural: thsnoen

3) Infinitivo: Usamos la forma del presente

Infinitivo $=$ Snó

4) Imperativo:

Se forma con los sufijos: Snó, Snóem, Snóemt

5) Cuando aprendas verbos por tu cuenta puedes preguntarlos por la primera persona, así sabrás sacar la raíz y conjugarlo entero.

6) Como regla general podemos decir que los verbos en másigt acaban en vocal breve (o-e) (ver aspectos fonéticos).

7) Verbos irregulares: Los dos más importantes son ghari (tener) y ackaîe (estar).

a) Ghari $=$ tener 
Presente
1) Ghari
4) Gharna
2) Ighac
5) Gharsen
3) Ghas
6) Gharsen

El pasado y el futuro se forman con togha y ad respectivamente.

b) Ackaie $=$ estar

Presente

1) Ackaie 5.1.) Ackáum

2) Ackash 5.2.) Ackáunt

3) Ackad 6.1.) Ackain

4) Ackanog 6.2.) Ackáint

El futuro se forma añadiendo ad- a la forma del presente.

c) El verbo "ser" no existe ni en presente ni en futuro. Para el pasado se utiliza el pasado del verbo ackaie.

G) La oración negativa:

1) Se utiliza el esquema $u a+$ verbo + sha Ejemplo: Ua ghari sha = uagharishá $=$ no tengo

2) Cuando el verbo en negativo va seguido de complemento directo se omite sha.

Ejemplo: Ua ghari ids $=$ uaghari $i d s=$ no tengo sueño.

3) Si el verbo está en pasado (con togha-) o en futuro, el prefijo se coloca antes de la partícula de negación ua

Ejemplo:

toghaghari $=$ tenfa

togha-ua-ghari = toghauaghari $=$ no tenía.

adaármoj = avisaré

$a d-u a-a a ́ r m o j=a d u a a ́ r m o j=$ no avisaré.

4) La negación de los adverbios se realiza según el esquema: 
J.A. Valverde, Halifa Kaddur, Yamila Mohamed

uashib + adverbio

Ejemplo: uashib + droçt $=$ uashibdroçt $=$ no poco.

I) La oración interrogativa.

1) Toda oración interrogativa sigue el esquema mint + verbo. Ejemplo: egued $=$ haces; $¿$ mintegued $?$ = ¿qué haces?

J) La oración exclamativa

1) La entonación y el uso de signos sin equivalentes a los españoles.

Ejemplo: ;árajhe dda! = ¡ven aquí!

2) La exclamación joh! se usa mucho en másigt ( $A$ !), delante del sujeto.

Ejemplo: ¡A Mojhémmed!, ¿Mámesh zétyid? = ¡eh, Mohamed!, ¿cómo estás?

K) Presente activo:

1) Variedad del verbo que en español puede traducirse por estar mas gerundio. El esquema es:

akat + verbo comiendo.

Ejemplo: Nesh + thittoj = yo como; nesh akatthittoj = yo estoy

L) Pronombres personales:

$\begin{array}{ll}\text { Nesh = yo } & \text { Neshén = nosotros } \\ \text { Schec = tú (masculino) } & \text { Quinnen = vosotros } \\ \text { Shem = tú (femenino) } & \text { Quinnit = vosotras } \\ \text { Nittá = él } & \text { Nihni = ellos } \\ \text { Nittaz = ella } & \text { Nihntin = ellas }\end{array}$

M) Pronombres y adjetivos posesivos:

$\begin{array}{ll}\text { Inú }=\text { mío } & \text { Dahná }=\text { nuestro } \\ \text { Nesh }=\text { tuyo } & \text { Nesh }=\text { vuestro } \\ \text { Çen }=\text { suyo } & \text { Çen }=\text { suyo }\end{array}$


N) Preposiciones más usadas:

1) $I=$ para

2) Çaci = al lado de, delante de

ejemplo: çaci záddarz = al lado de la casa.

3) Ardúfag = detrás

ejemplo: ardúfag fus = detrás de la mano.

4) Zighua $=$ sobre, encima de

ejemplo: Zighua misa = encima de la mesa

5) Sueddei = debajo de

ejemplo: sueddei zaská $=$ debajo del techo

6) $E n=d e$

ejemplo: túmobi en Ahmed $=$ el coche de Ahmed

7) "Con" sólo existe en las siguientes formas:

Aquídec $=$ conmigo

Aquides $=$ contigo

Aquides $=$ consigo, con él

Aquidna $=$ con nosotros

Aquidsen $=$ con vosotros

Aquidsen $=$ con ellos

8) $D h i=$ en

Ejemplo: dhi zámorz = en el suelo, o, en la patria

9) Adágher $=$ dentro de

Ejemplo: adágher áfarran = dentro del homo

10) Barra = fuera

ejemplo: irohe barra! = ¡vete fuera!

11) $A r=a$, hacia

Ejemplo: ar Mrisht 0 ar Zamrisht = hacia Melilla 
J.A. Valverde, Halifa Kaddur, Yamila Mohamed

12) $B r a=\sin$

Ejemplo: ushei atai bra nagna $=$ deme un té sin hierbabuena

13) $C \zeta i=$ de, desde

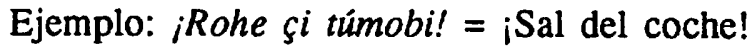

N) Conjunciones:

1) La más usada es de que significa "y"

ejemplo: Shec de nesh = tú (masculino) y yo

O) El artículo: en másigt no existe el artículo.

P) Adietivos demostrativos: Se suelen usar los siguientes:

1) Este / esta $=u a$

Ejemplo: Ariaçua $=$ este hombre

Záharantua $=$ esta niña

2) Ese $/$ esa $/$ aquel $/$ aquella $=$ uin

Ejemplo: shijed uin $=$ ese $/$ aquel papel

zámçida uín = esa $/$ aquella mezquita, o, ese $/$ aquel colegio

Q) Pronombres Relativos:
1) $u n n i=$ el que
2) inni $=$ los que
3) $z a n n i=$ la que
4) $z$ inni $=$ las que
5) lo que = jhuéix

Ejemplo: Shem eggued jhéix shem ejhsed $=$ tú $(\mathrm{fem}$.$) haces$ lo que quieres

R) Pronombres demostrativos:
1) $U a=$ éste
2) $i a=$ estos
3) $z a=$ ésta
4) zina $=$ éstas
5) $u i n=$ ése
6) $i n n i=$ ésos
7) zanni = ésa
8) zinni = ésas
9) jhuéix = esto

Ejemplo: ¿Uimi ijhsed aghe?. Zanni $=$ ¿Quién quiere leche?.Esa 
S) Pronombres personales como objeto directo:

1) Me: $i$, inú

Ejemplo: Ushei $=$ ushe $-i=$ Dame

2) Te: c, mo o más frecuentemente shec o shem

3) Le: nittá (masc), nittaz (femenino)

Ejemplo: ushe nittá = dale (a él)

4) Nos: dahná, neshén

5) Vos: Quinnen (masc.), quinnit (femenino)

(femenino)

6) Les = sent $($ masc. $)$, izsent $($ fem. $), 0$, nihni (masc. $),$ nihentin

7) Ahora bien, si pretendemos decir "te voy a" el "te" se integra en el prefijo ash (masculino) o ara (femenino)

Ejemplo: ash - aármoj = ashaármoj = te voy a avisar

T) Números:

$$
\begin{aligned}
& \text { una - ixet } \\
& \text { uno - ixen, uajhed } \\
& \text { dos - nain } \\
& \text { tres - threta } \\
& \text { cuatro - arba } \\
& \text { cinco - jhamstá } \\
& \text { seis - setta } \\
& \text { siete - sebaa } \\
& \text { ocho - zíminia } \\
& \text { nueve - tsâa } \\
& \text { diez - ashira } \\
& \text { once - jhedash } \\
& \text { doce - zenash } \\
& \text { trece - thretash } \\
& \text { catorce - arbatash }
\end{aligned}
$$


J.A. Valverde, Halifa Kaddur, Yamila Mohamed

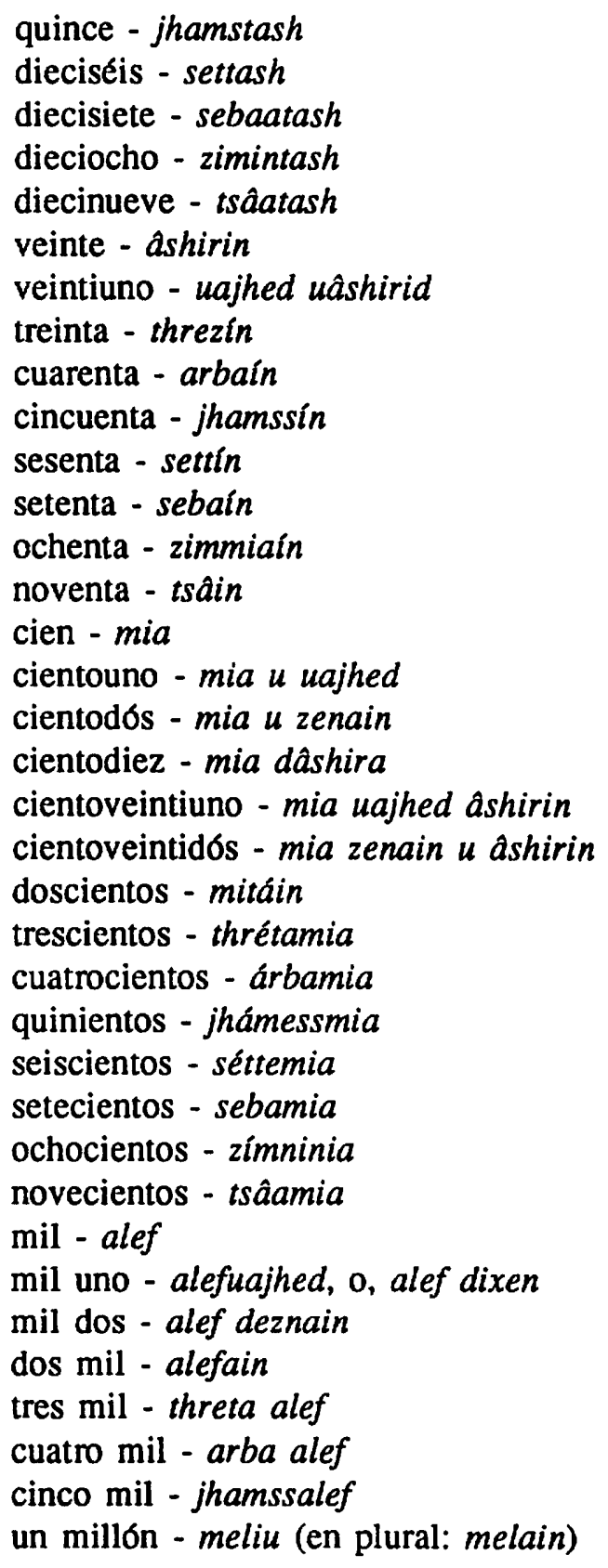

U) Ordinales: en másigt se emplean para los adjetivos 
ordinales, en general, las mismas palabras que designan los números, excepto:
1) Primero = ámeçuar
2) Primera = zámeçuar
II. Aspectos fonéticos:

Hasta ahora, lector, has podido leer un esquema gramatical que memorizarás con la práctica oral que te va a posibilitar el vocabulario que al final te ofrecemos. Ahora, antes de la práctica, conoce los aspectos fonéticos del másigt, necesarios para la práctica oral.

hombre.

1) “ $A, a$ ". Se trata de una "a" normal. Ejemplo: áriaç =

2) "Â, $\hat{a}$ ". Es una "a" gutural, que se pronuncia diciendo "ga" y suavizando la "g" hasta que casi desaparezca. Es una vocal sonora palatal. Ejemplo: â̂d = aún.

3) " $B, b$ ". Como la "b" española, es una consonante bilabial oclusiva sonora. Ejemplo: barra $=$ fuera.

4) " $C, c$ ". Se emplea igual que la "c" española. Puede ser velar oclusiva sorda (como en casa) o fricativa interdental sonora (como en cera). Ejemplo: Shec $=$ tú (masculino).

5) " $D, d$ ". Como la "d" española, se trata de una consonante dental oclusiva sonora. Ejemplo: dien = alli.

6) "Dh, dh". Se pronuncia como una "d" seguida de una " $\mathrm{z}$ ", es interdental fricativa sonora. Ejemplo: $A f r a d h=$ basura.

$=$ juramos

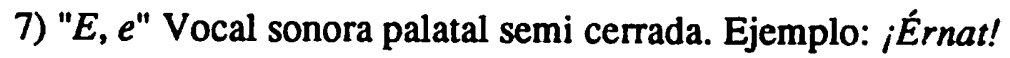

8) “ $F, f$ '. Como la " $\mathrm{f}$ " española, es una consonante labiodental fricativa sorda. Ejemplo: Áfarran $=$ horno. 
J.A. Valverde, Halifa Kaddur, Yamila Mohamed

9) " $G, g$ ". Como la " $g$ " española puede ser velar fricativa (Jorge) o velar oclutiva sonora (garra). Ejemplo: Zeguri $=$ estudios

10) "Gh, gh". Se pronuncia como "g" en garra seguida de una " $\mathrm{j}$ " . Es velar fricativa sonora. Ejemplo: aghe = leche

11) " $H, h$ ". Se trata de una hache aspirada como en el inglés "home" o en el andaluz "jarra" que se transforma en "harra", siendo esa $h$ una "j" suavemente pronunciada. Es velar sorda. Ejemplo: ¡árahad! = ¡ven!

12) "I, $i$ ". Vocal sonora palatal. Ejemplo: $u i m i=$ quien.

13) ' $J, j$ ". Se pronuncia igual que en el español. Se trata de una consonante velar fricativa sorda. Ejemplo: çímmej = puedo.

14) " $J h, j h$ ". Se trata de una hache aspirada pero más fuerte. Es el resultado de pronunciar una hache aspirada con el doble de intensidad. Ejemplo: ajhuri = carnero, gafe.

15) " $K, k$ ". No es coml la " $k$ " española. Se trata de un sonido resultante de pronunciar una " $k$ " seguida de una " $j$ " resultando unsonido velar africado sordo. Ejemplo: $̧ u k=$ zoco.

16) " $L, l$,. Al igual que nuestra "l", se trata de un sonido alveolar lateral sonoro. Es raro encontrarla sola, suele ir compuesta.

17) " $L l, l l$ ". No es nuestro sonido "ll", se trata de pronunciar dos "l" seguidas $(1+1)$ como en el italiano "Caracalla". Ejemplo: Allah = Alá, el nombre de Dios.

18) " $L h, l h "$. Se trata de nuestra "ll", es decir, un sonido palatal lateral sonoro. Elemplo: alhorred $=$ arrastras.

$=$ donde.

19) " $M, m$ ". Consonante alveolar nasal sonora. Ejemplo: meni 20) " $N, n$ ". Consonante alveolar nasal sonora. Ejemplo: anu $=$ pozo. 

= agujero.

21) " $O, o$ ". Vocal sonora velar semicerrada. Ejemplo: nokeb

22) " $P, p$ ". Igual que nuestra " $p$ " es un sonido raro en másigt y suele localizarse en palabras españolas. Se trata de un sonido bilabial oclusivo sordo. Ejemplo: ekampo = el campo.

23) " $Q, q$ ". Sonido velar oclusivo sordo, se emplea exactamente igual que en español. Ejemplo: quinneu = vosotros.

24) " $R, r$ ". Esta " $r$ " se emplea como si la pronunciásemos en medio de una palabra española como, por ejemplo, pera. Siempre se pronuncia así. Es un sonido alveolar vibrante sonoro. Si queremos realizar un sonido vibrante múltiple ("rr" en español en perro), escribiremos una doble "rr" ("rr"). Ejemplos: raxe = espera, afarran = horno.

25) " $S, s$ ". Al igual que en el español, se trata de un sonido alveolar fricativo sordo. Ejemplo: fus = mano.

26) "Sh, sh". Se trata de una "ch" suave, como "shore" en inglés, o "coche" con acento andaluz. Se trata de un sonido palatal fricativo sordo. Ejemplo: ;shof! = atiende

27) " $\zeta, \varsigma^{\prime}$ ". Es una "s" muy suave, como en francés "poison". Ejemplo: $a c ̧ i=$ mitad.

28) " $T, t$ ". Como en español, se trata de un sonido dental oclusivo sordo. Ejemplo: toghai = era, estaba.

29) "Th, th". Es un sonido resultante de pronunciar una " $t$ " seguida de una " $z$ ". Es un sonido interdental oclusivo sordo. Ejemplo: themáçight $=$ bereber, másigt.

30) " $U, u$ ". Vocal sonora velar cerrada. Ejemplo: ualo = nada.

31) " $X, x$ ". No es como nuestra " $x$ ". Es el resultado de pronunciar el sonido "sh" seguido de la " $y$ " española en yunque. Es un sonido palatal fricativo sonoro. Ejemplo: axarre = borrego. 
32) " $Y, y$ ". Se pronuncia como nuestra " $y$ " en yunque, es decir, es un sonido palatal fricativo sonoro. Es poco frecuente en la masigta. Ejemplo: yetshe $=$ jura.

33) " $Z, z$ ". Igual que nuestra " $z$ ", es un sonido interdental fricativo sordo. Se emplea y escribe igual que en el español, Ejemplo: zámçida $=$ mezquita, colegio.

Las vocales "e" y "o" son siempre breves. Para que comprendas que es una vocal breve, realiza este sencillo ejercicio: en la palabra "cesa" pronuncia lentamente la primera silaba (ce) y la segunda después (sa). Posteriormente repite normalmente la primera sílaba y lentamente la segunda. Finalmente pronuncia en la primera sílaba normalmente la "c", rápido la "e", y pronuncia la segunda sílaba de forma normal. Te habrás dado cuenta de que la " $e$ " (vocal breve) apenas se pronuncia.

108 กิol.

Las reglas de acentuación serán las mismas que en el espa-

La diéresis la utilizaremos cuando la " $h$ " vaya precedida de una letra con la cual forme un sonido determinado.

Ejemplo: Si escribimos áthedhmed la "th" la leeremos según lo explicado en el punto 29 de la fonética. Pero si queremos leer una "e" aspirada detrás de la " $\mathrm{t}$ " escribiremos: áthedhmed = destruyes. Igual ocurre en eshed $=$ grueso, gordo.

\section{Vocabulario mínimo propuesto}

Antes de comenzar debemos aclarar que con una llamada están marcados los verbos y palabras irregulares.

a) Ghari $=$ Tener. $($ Ver 3.F.7)

$\begin{array}{ll}\text { Túmobi }=\text { coche } & \text { Zasaat }=\text { reloj } \\ \text { Mashina = máquina } & \text { Crid }=\text { casi } \\ \text { Sabon }=\text { jabón } & \text { Shan marrez = a veces } \\ \text { Nuáddar = gafas } & \text { Erhémmedh }=\text { pañuelo }\end{array}$




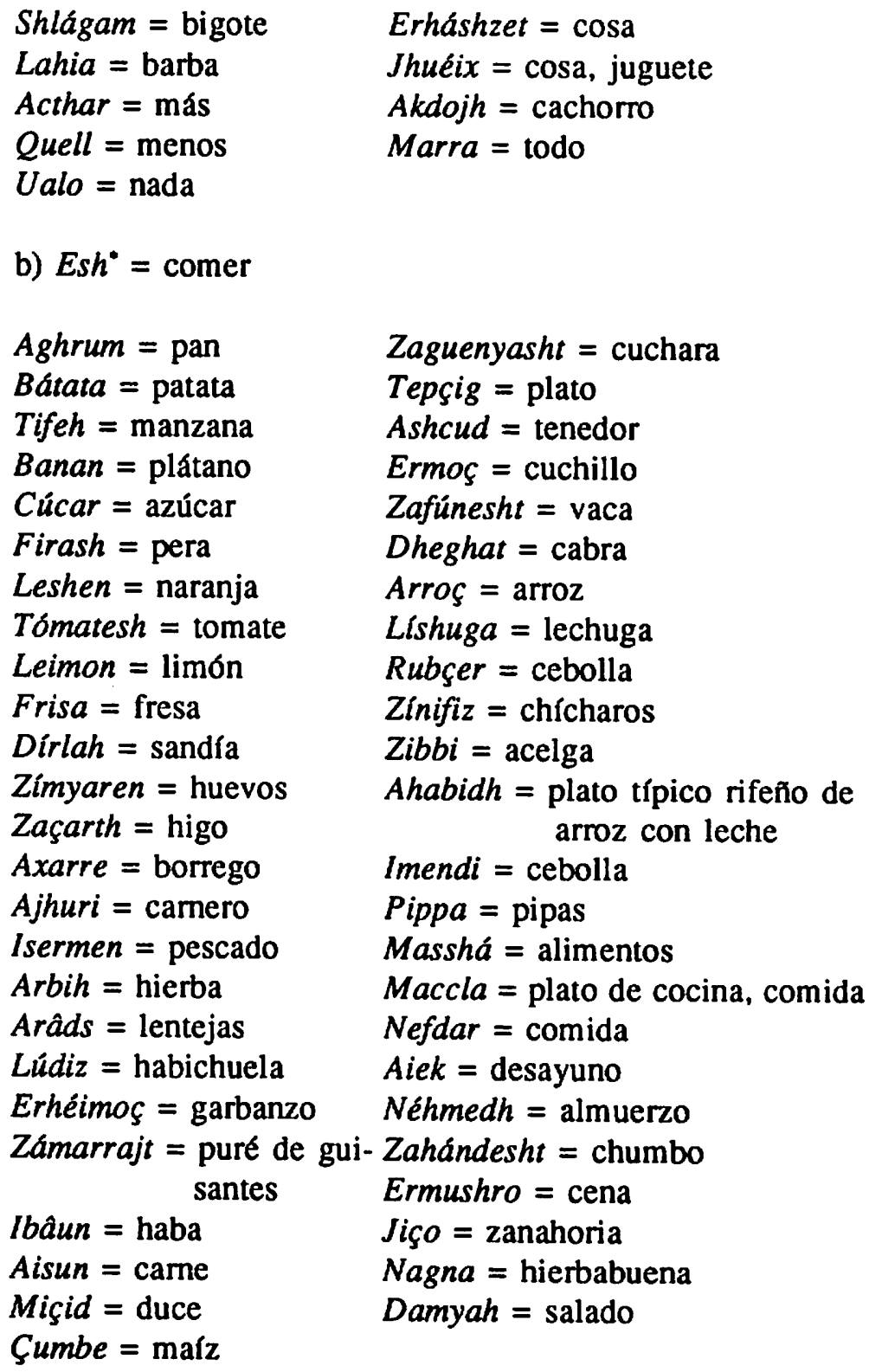

(*) Esh es irregular. El presente se forma con thittó, el pasado con shitó y el infinitivo e imperativo con esh. 
c) $\mathrm{Kem}^{*}=$ sentarse

$$
\begin{array}{ll}
\text { Dda }=\text { aquí } & \text { Dien }=\text { allí } \\
\text { Ammu }=\text { así } & \text { Iudesz }=\text { cerca } \\
\text { lucqush }=\text { lejos } & \text { Afusi }=\text { derecha } \\
\text { Dáserma }=\text { izquierda } & \text { Ardúfag }=\text { detrás } \\
\text { Çaci }=\text { delante, al lado de, } & \text { Monem }=\text { juntos } \\
\multicolumn{1}{c}{\text { junto a }} &
\end{array}
$$

d) Ehse (presente), Ehsite (pasado) = querer, amar

$$
\begin{array}{ll}
\text { Ajçí }=\text { perro } & \text { Attás = mucho } \\
\text { Droçt = poco } & \text { Shuei }=\text { poco } \\
\text { Zactof = hormiga } & \text { Isbah }=\text { bueno, buena } \\
\text { Esbajhod = guapo, guapa } & \text { Içi }=\text { mosca } \\
\text { Çiçua = avispa } & \text { Ibaâsh = bicho } \\
\text { Mush = gato } & \text { Elhob = amor } \\
& \\
\text { e) Çuó (presente), çue (pasado) }=\text { beber. }
\end{array}
$$

$$
\begin{array}{ll}
\text { Çumo }=\text { zumo } & \text { Emen }=\text { agua } \\
\text { Ercahuá }=\text { café } & \text { Atai }=\text { té } \\
\text { Aghe }=\text { leche } & \text { Shrá }=\text { alcohol (de bebida) } \\
\text { Çish }=\text { aceite } & \text { Erqueç = vaso }
\end{array}
$$

f) Adremde (presente), dremdé (pasado) = aprender.

$$
\begin{array}{ll}
\text { Themáçight }=\text { Másigt } & \text { Aspaniu }=\text { español } \\
\text { Âuer = palabra } & \text { Zámçida }=\text { colegio, mezquita } \\
\text { Ighuén = fácil } & \text { lughâar = difícil } \\
\text { Mojhállem }=\text { profesor, } & \text { Amhadar = alumno, discípulo } \\
\quad \text { maestro } & \text { Lemtiham }=\text { examen }
\end{array}
$$

g) Snó = saber, conocer

$$
\text { Mámesh }=\text { cómo, como } \quad \text { Mermi }=\text { cuándo, cuando }
$$

(") Ken es imperativo, "siéntate". se suele usar sólo de esta forma. 
Mághar $=$ por qué, porque $M e n i=$ dónde, donde

Marhan = cuánto, cuanto Shahar = cuánto cuesta

h) Dhaçmó = cerrar, bille (presente), blâe (pasado)

Zámorz = puerta

Nábira $=$ nevera

Ercuaçe $=$ ventana

i) Siauaro $=$ hablar

Dejia $=$ rápido

Sráquer $=$ lento

Eljarix $=$ extranjero

Errif $=$ Rif

Arrifi $=$ rifeño

j) Amné = creer (tener fe en)

Arbi $=$ Dios

Allah $=$ Dios

Mojhémmed $=$ Mahoma

Sohabi = profetas que estu-

vieron junto a Mahoma

Erjhámmem $=$ baño turco

Imsermen $=$ musulmanes

Tárib = almuedano

Erhix $=$ la Meca

k) Ezçré = ofr, escuchar

Musica $=$ música

Açfar = silbido

1) $E$ tçré $=$ ver, mirar

Zefusht $=$ sol

Shénnez $=$ cielo

Faut $=$ luz

Ighsar $=$ rfo

Agharrabud $=$ barco
Sidarbi $=$ El Señor

Sidnalsa $=$ Jesucristo

Nebi = el Profeta (Mahoma)

- Musa = Moisés

Zámçida = mezquita, colegio

Arume $=$ cristiano

Haram = pecado

Efquif = almuédano

Sheshterhix $=$ solideo

Ghanshó = cantar

lor = luna

Izren $=$ estrellas

Etram = oscuridad

Ebbehar $=$ mar

Zágharrabud = barca 
J.A. Valverde, Halifa Kaddur, Yamila Mohamed

m) Çimme $=$ poder
Imhed $=$ fuerte
$X a=$ fuerza
larhó $=$ débil
Iuâr $=$ violento
$I c ̧ a h=$ musculoso, recio

n) Atsghó = comprar, açinçó $=$ vender
Zimnia $=$ dinero
Zahant $=$ tienda
Iarheç $=$ barato
Ighrá = caro
n) $G a \hat{a} e=$ subir

Escaler $a=$ escalera

Tsanén $=$ cuesta

o) Ushé = dar

Çuden $=$ beso

Shuei $=$ poco

112

Sha $=$ algo

p) Verbos impersonales:
Adfer $=$ nieva
Ançar = llueve
Asino $=$ hay nubes
Enharisbah = hace buen tiempo
Enharueihere $=$ hace $\mathrm{mal}$
Açmed $=$ hace viento tiempo
Arhamó $=$ hace calor
$E c ̧ \bmod =$ hace frío
Dinne $=$ hay
q) Arre $=$ oler
Mlih = bien
Ueihere $=$ mal, malo, feo
Açarred $=$ pedo, ventosidad $I c ̧ e ́=$ peer
r) Sharhó = odiar
$\begin{array}{ll}\text { Damshun }=\text { golfo } & \text { Ueihere = malo } \\ \text { Yejha }=\text { "chorizo", ladron } & \text { Aghuan = ladrón } \\ \text { Ajhuri }=\text { gafe } & \end{array}$ 
s) $T e j h e d d m e ́$ = trabajar

$$
\begin{array}{rlrl}
\text { Adhbib }=\text { médico } & & \text { Mojhállem }=\text { profesor, maestro } \\
\text { Abhari }= & \text { campesino, } & & \text { Adisharçe }=\text { labrar } \\
\text { labrador } & & \text { Adiçó }=\text { sembrar }
\end{array}
$$

t) $T u c g u \delta=$ usar, llevar puesto

\begin{tabular}{|c|c|}
\hline Asdif $=$ cabeza & Ajhsho = vagina \\
\hline Zimmiuen $=$ cejas & Táhena = nalgas \\
\hline Zibiriuen $=$ pestañas & Endar $=$ pie \\
\hline Thitt $=0 j \mathrm{o}^{* *}$ & Dan = pierna \\
\hline Ança $=$ nariz & Ighatshen = brazos \\
\hline Ajmon $=$ boca & Dad $=$ dedo \\
\hline Zániarz $=$ frente & Zosoz $=$ tos \\
\hline Aghumbu = rostro & Agdiç = barriga \\
\hline Dheça = hígado & Estumaghu = estómago \\
\hline Acclau $=$ pene & Abrur $=$ pene \\
\hline$U r=$ corazón & Igheç $=$ hueso \\
\hline $\operatorname{Irç}=$ lengua & Idemen $=$ pecho \\
\hline Çumbe $=$ estreñimiento & Rouxa = diarrea \\
\hline Eddua $=$ medicina & Eddua zaçmod = curandería \\
\hline$F u s=\operatorname{mano}{ }^{* \ldots *}$ & $F u d h=$ rodilla \\
\hline
\end{tabular}

$\begin{array}{ll}\text { Nuáddar }=\text { gafas } & \text { Sáruar }=\text { pantalones } \\ \text { Jersi }=\text { jersey } & \text { Zájmixat }=\text { camisa } \\ \text { Ziçira = calzado } & \text { Erbot }=\text { bota } \\ \text { Harcuç = zapato } & \text { Zamquieçt }=\text { pulsera } \\ \begin{array}{l}\text { Zahádent }=\text { anillo } \\ \text { Ixib = bolsillo }\end{array} & \text { Funara }=\text { pañuelo de cabeza, } \\ \text { foulard }\end{array}$

u) Itecsaie $=$ doler

") Este verbo sólo se usa en esta forma y se traduce por: "me duele".

(") El plural de thitt es thittauen.

(0)) El plural de dad es idubdan

(*..) El plural de fus es ifasen. 
J.A. Valverde, Halifa Kaddur, Yamila Mohamed

v) Care $=$ decir

Acçar $=$ broma

Shof = exclamación que se traduce por ¡mira! en un claro préstamo del árabe.

w) Eddare = vivir

Zaddarz $=$ casa

Corçe $=$ silla

Ercuaçe $=$ ventana

Macra $=$ sartén

Púrtal $=$ portal

Meftá $=$ llave

Zaská $=$ techo

Mrisht, $o$, Zamrisht $=$ Melilla

Naddur $=$ Nador

Zahania $=$ techo

114

Amtárbadh $=$ especie de sofá sin respaldo

Remrah $=$ patio

x) Ittao $=$ volar

Ashdidh = pájaro

Zádbash $=$ avecilla

y) Samhae $=$ perdonar

acsar $=$ broma

z) Uaró = estar cansado

¡Eshfá! = ¡Ya basta!

Ibsred $=$ pesado, lerdo

z') $F a r h e ́=$ alegrarse
Mis $a=$ mesa

Záuorz = puerta

Erhrid $=$ pared

Nábira $=$ nevera

Axar $=$ vecino

Akbush $=$ jarra

Foque $=$ azotea

Aspania $=$ España

Áfarran = horno

Sufa $=$ sofá

Çif $=$ toalla

Bretermá = cuarto de baño

$A n u=$ pozo

Shénnez $=$ cielo

Sidi $=$ señor

¡Ska! $=$ ¡silencio!

Rojhod $=$ ahora 


\section{Algunas expresiones}

Munana, Allah munana $=$ por amor de Dios

Tira mâina $=$ sigue adelante

Barc Allah fic = muchas gracias

Asselam alicom = la paz sea contigo (con nosotros)

Esbarjher $=$ buenos días

Miserjher $=$ buenas tardes

¿Mámesh zétyid? $=$ ¿como estás?

$X e i$ tránker $=$ déjame tranquilo

In shae Allah $=$ si Dios quiere

$U a=$ sí

$L-l a ́=$ no

\section{Bibliografia consultada}

IBAÑEZ, Esteban. Diccionario español-rifeño. Madrid, 1944.

LAOUST, E. "Le dialecte berbére du rif". En: Hesperis, T. VII, $2^{\circ}$ trimestre, Rabat, 1927.

OUARIACHI, Kais-Marzouk. "Elèments pour la compréhension de la problematique tamazight". En: Awal: Cahiers d'etudes berbères, N. 4. París 1988.

PEREGRÍN PEREGRÍN, Ginés. Rudimentos de bereber rifeño. Tetuán 1944.

SARRIONANDÍA, Pedro. Gramática de la lengua rifeña, Tánger, 1905.

TAIFI, Miloud. "Problèmes mèthodologiques relatifs à la confection d'un dictionnaire du tamazight". En: Awal: Cahiers d'etudes berberes, N. 4, París, 1988.

Tasafut, N. 1 Rabat, 1991. 